\title{
Um link entre a resolução analítica e a abordagem computacional em uma aula de Equações Diferenciais Ordinárias (EDOs) para turmas do curso de Engenharia de Computação
}

\author{
Teodora Pinheiro Figueroa ${ }^{1}$ \\ Departamento de Matemática, UTFPR, Pato Branco, PR
}

\begin{abstract}
Resumo. Este trabalho tem como objetivo apresentar o resultado de uma investigação na disciplina de EDOs em uma turma de engenharia de computação. Essa investigação se refere a questões relacionadas a inserção de atividades de implementação computacional, como uma ferramenta de apoio, no sentido de estabelecer um link entre a disciplina de EDOs e uma área específica do curso de engenharia de computação: a engenharia de software, área em que o engenheiro da computação desenvolve programas computacionais e aplicativos para os mais diversos fins. Sendo assim, analisando a grade curricular do curso de engenharia de computação da Universidade Tecnológica Federal do Paraná, campus Pato Branco (UTFPR-PB) constatou-se que as disciplinas de Fundamentos de Programação e Lógica para Computação fazem parte do primeiro semestre. Então, de acordo com a grade curricular, os alunos deste curso têm pré-requisito para implementar os métodos estudados na disciplina de EDOs (disciplina do terceiro semestre). Sendo assim, decidiu-se fazer uma experiência investigativa fundamentada na Teoria das Situações Didáticas (TSD), a fim de responder as seguintes questões: É possível e viável fazer um link entre aspectos teóricos da disciplina de EDOs e a implementação computacional, ferramenta básica do curso de Engenharia de Computação? Quais competências e habilidades podem ser desenvolvidas e, o quanto esta metodologia pode contribuir ao aprendizado dos alunos? Qual o nível de satisfação dos alunos diante da possibilidade de se fazer este link? O processo investigativo proporcionou meios de responder à estas questões. Além disso, a análise através da TSD comprovou a viabilidade desta investigação principalmente no que diz respeito a evidenciar aspectos importantes no desenvolvimento de competências e habilidades específicas ao se fazer um link entre as disciplinas da área de matemática e da área de computação.

Palavras-chave. Equações Diferenciais Ordinárias, Teoria das Situações Didáticas, Engenharia de Computação, Implementação Computacional
\end{abstract}

\section{Introdução}

Várias pesquisas tem sido realizadas sobre o ensino e aprendizagem de EDOs, como por exemplo o trabalho de Oliveira e Igliori [4], o qual apresenta um levantamento bibliográfico de trabalhos realizados, no período de 2000 a 2011. Estes trabalhos propõem diversos tipos de metodologias diferenciadas a fim de amenizar as dificuldades dos alunos e proporcionar uma aprendizagem significativa.

Na UTFPR, campus Pato Branco, a disciplina de EDOs compõe o terceiro período (semestre) dos cursos de engenharia. As aulas são teóricas e, a importância é dada a resolução analitica das

\footnotetext{
${ }^{1}$ teodora.pinheiro@gmail.com
} 
EDOs. A resolução analítica é algo de extrema importância e, essencial no que diz respeito ao domínio da parte algébrica e, escrita matemática. Porém, a valorização do enfoque algébrico sobre o gráfico e o numérico na resolução de problemas-padrão tem sido alvo de reflexão e, apontado por Dullius et al [3]. Acredita-se que em um curso de engenharia de computação, foco deste trabalho, principalmente a parte numérica pode ser mais explorada, como por exemplo, na forma de uma atividade em listas de exercícios. Pois, o aluno do curso de engenharia de computação possui um diferencial em relação aos alunos de outros cursos, ou seja, tem uma preferência por ferramentas computacionais.

Sendo assim, sentiu-se a necessidade de se fazer algumas investigações a fim de tentar responder as seguintes questões:

Questão 01: É possível e viável fazer um link entre aspectos teóricos da disciplina de EDOs e a implementação computacional, ferramenta básica do curso de Engenharia de Computação?

Questão 02: Quais competências e habilidades podem ser desenvolvidas e, o quanto esta metodologia pode contribuir ao aprendizado dos alunos?

Questão 03: Qual o nível de satisfação dos alunos diante da possibilidade de se fazer este link?

A fim de responder a Questão 01 fez-se uma pesquisa na grade curricular do curso de Engenharia de Computação da UTFPR. E, observou-se que o primeiro semestre é constituido pela disciplina de Fundamentos de Programação (FP), a qual fornece elementos básicos de uma linguagem de programação estruturada e Lógica para Computação (LC), a qual tem como um dos objetivos desenvolver o raciocínio abstrato utilizado em programação de computadores. Logo, constatou-se a viabilidade do ponto de vista de pré-requisito necessário à inserção da parte numérica através da implementação computacional na disciplina de EDOs. Mas, surgiu uma outra questão referente aos alunos não aprovados nas disicplinas de FP e LC. Neste caso, os mesmos teriam a oportunidade de rever alguns conceitos e interagir com os outros colegas a fim de sanar algumas dificuldades existentes.

Sendo assim, decidiu-se dar continuidade ao processo investigativo a fim de responder as Questões 02 e 03. Para isso, inseriu-se no planejamento da disciplina duas aulas no laboratório de informática sobre o tópico de EDOs lineares de primeira ordem. Os detalhes desta experiência serão relatados nas próximas seções.

A metodologia em sala de aula foi planejada com foco na Teoria das Situações Didáticas (TSD) de Brousseau [2], onde o aluno é o sujeito ativo que interage com o meio e, o professor assume o papel de mediador, observador em cada etapa das dialéticas de ação (diante do problema proposto sem a intervenção do professor), formulação (de suas hipóteses), validação (a partir dos seus registros e argumentações ) e institucionalização (institucionalização do saber realizada pelo professor).

\section{Equações Diferenciais Ordinárias Lineares de Primeira Ordem}

As Equações Diferenciais Ordinárias Lineares são equações que podem ser escritas da seguinte forma:

$$
a_{n}(t) \frac{d^{n} y}{d t^{n}}+a_{n-1}(t) \frac{d^{n-1} y}{d t^{n-1}}+\ldots+a_{1}(t) \frac{d y}{d t}+a_{0}(t) y=g(t)
$$

O foco deste trabalho são as Equações Diferenciais Ordinárias Lineares de primeira ordem:

$$
a_{1}(t) \frac{d y}{d t}+a_{0}(t) y=g(t)
$$

Dividindo ambos os lados da equação por $a_{1}(t), a_{1}(t) \neq 0$ temos: 


$$
\frac{d y}{d t}+p(t) y=q(t)
$$

onde $p(t)=\frac{a_{0}(t)}{a_{1}(t)}$ e $q(t)=\frac{g(t)}{a_{1}(t)}$

A equação 3 recebe o nome de Equação Diferencial Ordinária (EDO) Linear Homogênea se $q(t) \equiv 0$. Caso contrário recebe o nome de EDO linear Não-Homogênea.

Para as Equações Diferenciais Ordinárias Lineares Homogêneas (EDOLH) de primeira ordem a resolução analítica pode ser realizada usando Variáveis Separáveis [5]. E, para as Equações Diferenciais Ordinárias Lineares Não-Homogêneas (EDOLNH) de primeira ordem há a necessidade de um fator integrante. Todos os detalhes de resolução podem ser encontrados em [5].

Há vários métodos que resolvem analiticamente uma EDO; mas, é importante deixar claro para os alunos de graduação que nem sempre é possível obter uma solução analítica.

\section{Metodologia}

O planejamento das aulas sobre EDOs lineares de primeira ordem foi disposto conforme a Tabela 1:

Tabela 1: Planejamento das Aulas

\begin{tabular}{|c|c|c|}
\hline Aulas & Conteúdo & Local \\
\hline $01(50$ minutos $)$ & EDOLH primeira ordem & sala de aula \\
\hline $02(50$ minutos $)$ & EDOLNH primeira ordem & sala de aula \\
\hline $03(50$ minutos $)$ & Algoritmo Computacional & laboratório de informática \\
\hline $04(50$ minutos $)$ & Implementação Computacional & laboratório de informática \\
\hline
\end{tabular}

Este trabalho foi aplicado em uma turma que era inicialmente composta por 30 alunos, sendo que 09 alunos desistiram do curso e, nesta aula em especifico faltaram 02 alunos.

As situações didáticas foram desenvolvidas na forma de uma conversa entre os alunos e a professora. Pois acredita-se que é apenas através do diálogo e de suas respectivas "pausas"que os alunos conseguem assimilar os detalhes existentes em cada tópico abordado. E esta visão é fundamentada pelo construtivismo didático, o qual dá ênfase à dimensão social na aquisição dos conhecimentos. Segundo Almouloud [1] os processos de aquisição do conhecimento não são unicamente situados do lado dos sujeitos individualmente, mas da classe; a aquisição deve ser o resultado de um processo de adaptação dos sujeitos às situações que o professor organizou, nas quais as interações com os outros alunos terão um papel importante.

A matemática, assim como qualquer ciência é rica em detalhes, que precisam ser observados e explorados a partir de situações didáticas apropriadas para este fim. Sendo assim, cabe ao professor mediar este processo de busca e exploração. Pois quando o professor comenta sobre os detalhes, impede o aluno de desenvolver as suas competências e habilidades. Brousseau [2] defende que a aquisição dos conhecimentos depende, em primeiro lugar, das características das situações nas quais os sujeitos são colocados.

As situações didáticas que serão apresentadas referem-se a Aula 03, conforme a Tabela 1. A Aula 03 tem como objetivo mostar que é possível estabelecer um link entre aspectos teóricos (Aulas 01 e 02) e a implementação computacional. E, dessa forma procurar respostas à Questão 01 e Questão 02, as quais fazem parte do processo investigativo deste trabalho.

Situação I: Dada uma equação diferencial $y^{\prime}=f(t, y)$ como encontrar a função y que satisfaz esta equação diferencial? 
Situação II: Qual a característica comum entre a resolução analítica de uma EDOLH e uma EDOLNH?

Situação III:

Definição 3.1. Considerem-se $m$ subintervalos de $[a, b],(m \geq 1)$, e seja $x_{j}=x_{0}+j_{h}$ onde $h=\frac{b-a}{m}, j=0, \ldots, m$ e $x_{j} \in[a, b]$. Ao conjunto $I_{h}=\left\{x_{0}, x_{1}, \ldots, x_{m}\right\}$ obtido da forma anterior chama-se rede ou malha de $[a, b]$.

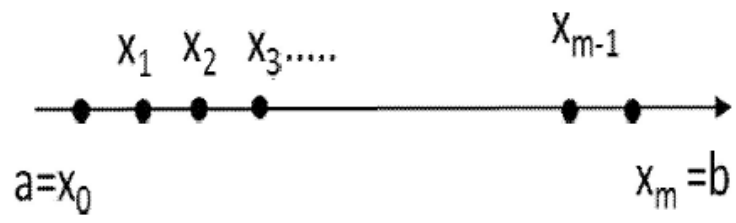

Figura 1: Malha.

A Figura 1 ilustra a definição 3.1 e, contribui para a resolução do ponto de vista da implementação computacional do Problema de Valor Inicial (PVI), Equação 4:

$$
\left\{\begin{array}{l}
y^{\prime}(t)=f(t, y(t)) \\
y\left(t_{0}\right)=y_{0}=\eta, \eta \quad d a d o .
\end{array}\right.
$$

o qual consiste do cálculo das aproximações $y_{1}, y_{2}, \ldots, y_{m}$ para as soluções exatas $y\left(x_{1}\right), y\left(x_{2}\right), \ldots, y\left(x_{m}\right)$. Logo, a partir do conceito de malha como obter um algoritmo computacional para a resolução do PVI (Equação 4)? Que relação pode ser estabelecida com o método de resolução analítica?

Situação IV: Encontre a solução computacional para o PVI

$$
\left\{\begin{array}{l}
y^{\prime}=t-y+2 \\
y(0)=2
\end{array}\right.
$$

Utilize a linguagem computacional de sua preferência.

\section{Resultados}

Nesta seção são apresentadas algumas observações em relação as respostas dos alunos referente as Situações da seção anterior. Cada Situação é caracterizada pelas dialéticas de Ação, Formulação, Validação e Institucionalização.

Na Situação I, a maioria dos alunos afirmaram que a resolução da EDO seria dada através da integração. Na Situação II, eles tiveram um pouco de dificuldade e, levou tempo, a partir de intervenções do professor para que eles percebessem que o processo é o mesmo e, por isso a importância do fator integrante. Na Situação III os alunos comentaram sobre o processo de integração, mas não sabiam validar a sua resposta. Faltou refletir mais sobre o significado de integração, ou seja, de conceitos do cálculo diferencial e integral.

E, por fim esta situação precisou ser mais detalhada para que o objetivo fosse atingido, ou seja, para que eles conseguissem desenvolver a resposta dada de que envolve um processo de integração, ou melhor para que eles conseguissem expressar matematicamente a resolução numérica do PVI (Equação 4). Segue uma parte do diálogo entre os alunos e a professora:

Professora: Basta integrar. Mas, como integrar $y^{\prime}(t)=f(t, y(t))$ ? 
Após uma pausa a professora prossegue com outra pergunta:

Professora: Lembram-se da nossa malha? Ver Figura 1. Queremos as aproximações das soluções $y_{1}, y_{2}, \ldots, y_{m}$, como vocês disseram precisamos integrar. Mas, qual intervalo de integração?

Aluno: Ah...integrar nesta malha.

Professora: Certo! Tentem expressar como.

Aluno: A cada dois pontos.

Professora: Dê um exemplo.

Aluno: Entre cada dois pontos, $x_{0}$ e $x_{1}, x_{1}$ e $x_{2} \ldots$

Logo, escreveu-se a Equação 6 no quadro, resultado da discussão com os alunos referente ao processo de integração de $y^{\prime}(t)=f(t, y(t))$ entre $x_{j}$ e $x_{j+1}$ :

$$
y\left(x_{j+1}\right)-y\left(x_{j}\right)=\int_{x_{j}}^{x_{j+1}} f(t, y(t)) d t
$$

Professora: Pessoal, integramos e, é possível expressar esta integral de outra forma?

Alunos: Sim.

Professora: Então como podemos aproximar esta integral? Em que consiste o processo de integração?

Aluno: a integral dá a área abaixo da curva.

Professora: Muito bem. Logo, como resolver isso? Como podemos expressar esta integral como área abaixo da curva?

Aluno: A base é o intervalo e a altura a função.

Professora: Isso mesmo. Logo, podemos expressar de forma grosseira que a base é: $\mathrm{h}=$ tamanho do intervalo $=x_{j+h}-x_{j}$ e , a altura dada pela função: $f\left(x_{j}, y_{j}\right)$. Fazendo essa substituição na Equação 6, a mesma torna-se uma fórmula recursiva que pode ser implementada computacionalmente.

$$
y_{j+1}=y_{j}+h f\left(x_{j}, y_{j}\right), j=0,1, \ldots, m-1
$$

Dessa forma, complementamos a resposta da Questão 01, de que é possível estabelecer um link entre a resolução numérica e a resolução analítica, pois se analisarmos os detalhes, o processo de resolução é o mesmo. E, para entender a resolução do PVI do ponto de vista das aproximações da função em cada $x_{j}, \quad j=0,1, \ldots, m-1$, foi preciso ter entendimento do processo de integração.

Com relação a resposta para a Questão 02 verificou-se que a metodologia baseada na TSD proporciona aos alunos mais autonomia para desenvolver as suas competências e habilidades a partir das interações entre o aluno, o saber e o meio de interação (atividade proposta em casa situação didática). Neste caso, em específico as competências e habilidades referem-se a interpretação da linguagem matemática do ponto de vista analítico e, a ampliação desta leitura e interpretação para o ponto de vista da abordagem computacional.

Na Situação IV, Aula 04, os alunos realizaram a implementação computacional em linguagem C, partindo da Equação 7 para a resolução do PVI (Equação 5).

Com relação a Questão 03 o nível de satisfação dos alunos foi de 100\%. Seguem dois depoimentos conforme as Figuras 2 e 3: 


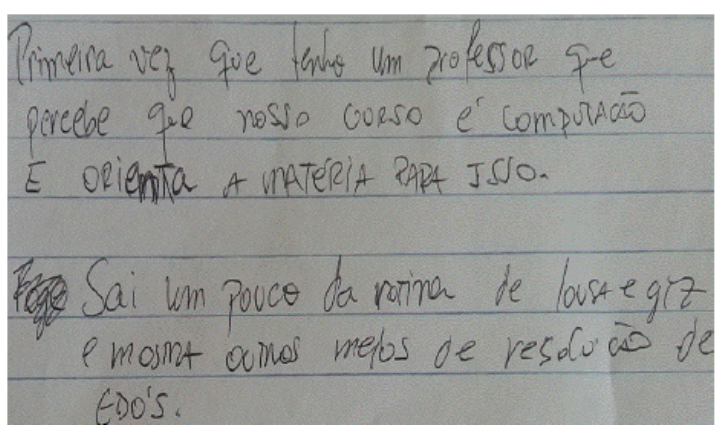

Figura 2: Depoimento 1.

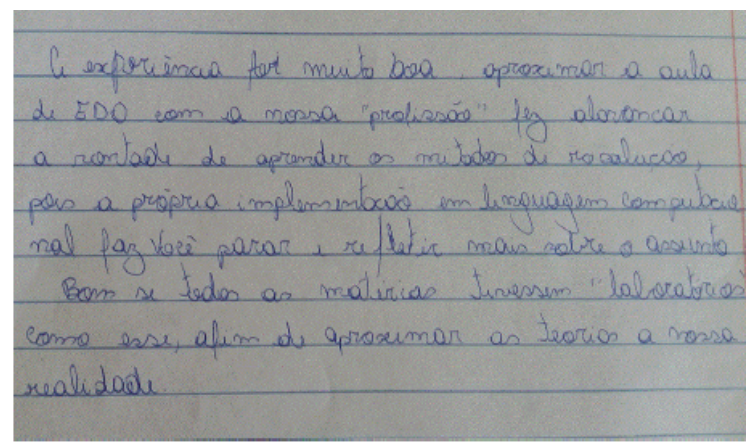

Figura 3: Depoimento 2.

\section{Conclusões}

As questões de investigação deste trabalho foram contempladas no decorrer de seu desenvolvimento e, o nível de satisfação dos alunos, conforme relatado nas Figuras 2 e 3 é algo que motiva a dar continuidade a essa investigação com outros tópicos da disciplina de EDOs.

A aproximação das disciplinas da área de matemática com a área de formação do aluno é algo a se pensar, principalmente no sentido de provocar questões que tenham como objetivo estabelecer conexões entre a matemática e uma área específica do curso.

A familiaridade dos alunos com os algoritmos computacionais, estudados nas disciplinas de FP e LC, favoreceu o entendimento do conceito de malha e, consequentemente, o entendimento da abordagem computacional. É importante deixar claro que não foram abordadas questões sobre a propagação de erro na fase de resolução numérica, conteúdo da disciplina de Cálculo Numérico.

A metodologia proposta com foco na Teoria das Situações Didáticas foi de extrema importância para o professor fazer o exercício de estabelecer as pausas necessárias de acordo com as dialéticas de ação, formulação, validação e institucionalização de forma a contribuir para a aprendizagem do aluno. É preciso dar a oportunidade aos alunos pensarem nos detalhes, que na maioria das vezes para o professor são óbvios. De acordo com esta teoria o professor assume o papel de observador e/ou mediador durante os momentos das dialéticas em cada situação didática proposta.

Pode-se conluir que os resultados foram satisfatórios tanto em relação ao aprendizado quanto ao interesse e satisfação dos alunos diante da possibilidade de fazer um link entre a disciplina e uma ferramenta específica do seu curso: a implementação computacional. 


\section{Referências}

[1] S. A. Almouloud. Fundamentos da didática da matemática. UFPR, Curitiba, 2007.

[2] G. Brousseau. Introdução ao estudo da teoria das situações didáticas: conteúdos e métodos de ensino. Ática, São Paulo, 2008.

[3] M. M. Dullius, E. A. Veit, I. S. Araujo. Uso de recursos Computacionais para o ensino e aprendizagem de Equações Diferenciais. In Anais do Congresso Brasileiro de Educação em Engenharia (XXXV COBENGE), Curitiba, Paraná, Brasil, 2007.

[4] E. A. Oliveira, S. B. C. Igliori. Ensino e Aprendizagem de Equações Diferenciais: um levantamento preliminar da produção científica. EM TEIA - Revista de Educação Matemática e Tecnológica Iberoamericana, volume 4, número 2, 2013.

[5] D. G. Zill, M. R. Cullen. Equações Diferenciais. Pearson Education do Brasil, São Paulo, 2008. 